An Elliptic Problem Arising From the Unsteady Transonic Small Disturbance Equations

Sunčica Canić ${ }^{*}$ Barbara Lee Keyfitz**

Research Report UH/MD - 171

* Mathematics Department, Iowa State University

**Department of Mathematics, University of Houston 


\title{
An Elliptic Problem Arising From the Unsteady Transonic Small Disturbance Equation*
}

\author{
Sunčica Čanić ${ }^{\dagger}$ \\ Mathematics Department \\ Iowa State University
}

\author{
Barbara Lee Keyfitz \\ Department of Mathematics \\ University of Houston
}

September 14, 1995

\begin{abstract}
Abstreact
We prove a theorem on existence of a weak solution of the Dirichlet problem for a quasilinear elliptic equation with a degeneracy on one part of the boundary. The degeneracy is of a type ("Keldysh type") associated with singular behavior - blow-up of a derivative at the boundary. We define an associated operator which is continuous, pseudo-monotone and coercive and show that a weak solution displaying singular behavior at the boundary exists.
\end{abstract}

\section{Contents}

1 Introduction

* Journal of Differential Equations, to appear.

ISupported by the Texas Advanced Research Program under Grant 003652-124-ARP. Support by the Fields Institute is also acknowledged.

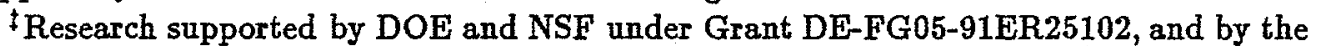
Texas Advanced Research Program under Grant 003652-124-ARP. Support by the Fields Institute is also acknowledged. 


\section{DISCLAIMER}

This report was prepared as an account of work sponsored by an agency of the United States Government. Neither the United States Government nor any agency thereof, nor any of their employees, make any warranty, express or implied, or assumes any legal liability or responsibility for the accuracy, completeness, or usefulness of any information, apparatus, product, or process disclosed, or represents that its use would not infringe privately owned rights. Reference herein to any specific commercial product, process, or service by trade name, trademark, manufacturer, or otherwise does not necessarily constitute or imply its endorsement, recommendation, or favoring by the United States Government or any agency thereof. The views and opinions of authors expressed herein do not necessarily state or reflect those of the United States Government or any agency thereof. 


\section{DISCLAIMER}

Portions of this document may be illegible in electronic image products. Images are produced from the best available original document. 
2 The Degenerate Boundary and the Comparison Principle 6

2.1 Singularities in the Solution $\ldots \ldots \ldots \ldots \ldots \ldots$

2.2 The Comparison Principle . . . . . . . . . . 7

3 A Linear Model Equation 8

3.1 Existence of a Weak Solution in a Hilbert Space . . . . . 9 9

4 The Weighted Sobolev Space Setting 11

4.1 Modification of the Problem . . . . . . . . . . . 11

4.2 Embeddings of the Hilbert Space $\mathcal{H}_{\alpha} \ldots \ldots \ldots \ldots$

4.3 Weak Formulation of the Modified Problem . . . . . . . 16

5 Existence of a Weak Solution 19

5.1 Properties of $T \ldots \ldots \ldots \ldots \ldots \ldots \ldots$

5.2 Bounds on the Weak Solution $\ldots \ldots \ldots \ldots \ldots$

6 Conclusions 26

A Appendix: Compactness of the Embedding 27

\section{Introduction}

The following Dirichlet boundary value problem for a quasilinear degenerate elliptic equation arises in studying self-similar solutions of hyperbolic conservation laws in two space dimensions. The equation is

$$
\left((u-\rho) u_{\rho}+\frac{u}{2}\right)_{\rho}+u_{\eta \eta}=0,
$$

in the bounded domain $\Omega$ shown in Figure 1 . The boundary data is given by $u=g$ on $\partial \Omega$, where $g=\rho_{m}$ on the degenerate boundary $\Gamma_{2}$ and on $\Gamma_{\infty}$, and $g=\tilde{g}$ on $\Gamma_{3}$, where $\tilde{g} \geq \rho_{m}$ is continuous and symmetric with respect to the $\rho$-axis.

Equation (1) results from scaling variables $\left(\xi=x / t, \eta=y / t, \rho=\xi+\eta^{2} / 4\right)$ in the unsteady transonic small disturbance (TSD) equation, also called the 2-D Burgers equation or Zabolotskaya-Khoklov equation:

$$
\begin{array}{r}
u_{t}+u u_{x}+v_{y}=0, \\
-v_{x}+u_{y}=0 .
\end{array}
$$




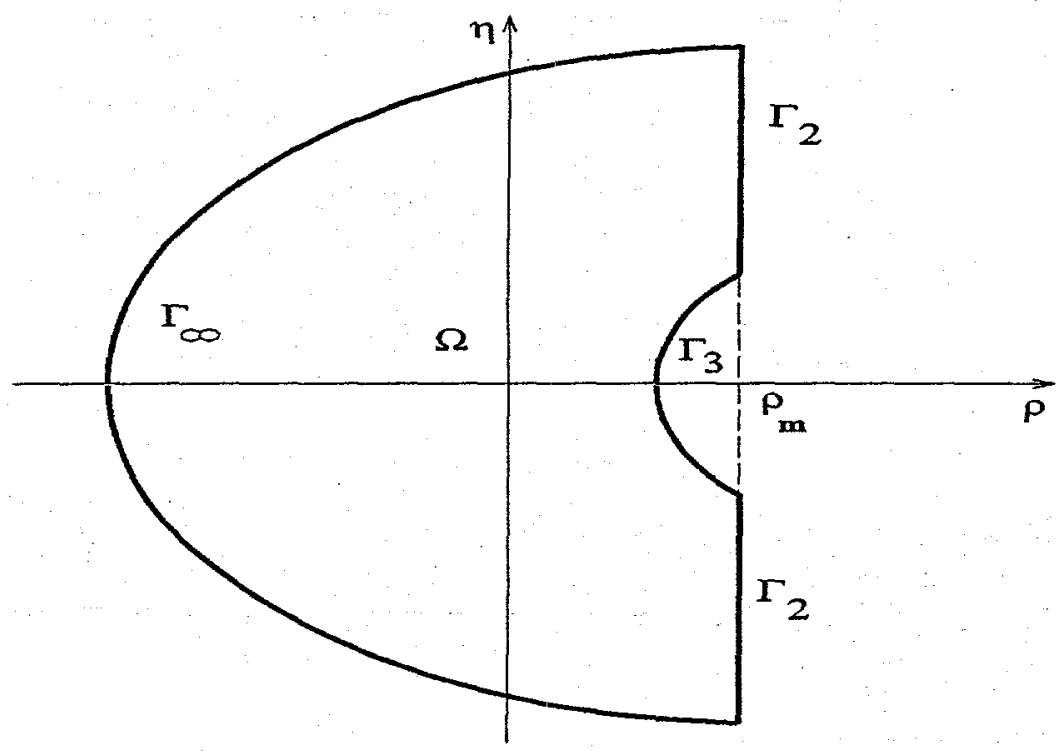

Figure 1: Domain and Boundary Components of the Dirichlet Problem

Reasons for this scaling are discussed in [3]. The system of equations (2) has been derived and used by several authors (see [7], [14] and the references therein) to model problems in nonlinear acoustics and nonlinear geometrical optics, and also in unsteady transonic flow. Brio and Hunter in [2] use (2) as an asymptotic reduction of the Euler equations in order to study weak shock reflection. The system (2) retains the nonlinear wave structure of the gas dynamics equations in the form of a particularly simple nonlinearity, and may give a description of the transition between regular and Mach reflection for weak shocks. This phenomenon has been extensively studied numerically and experimentally (see, for example, [5], [15]); however, there are only a few related analytical results. We mention Morawetz's study of a full-potentialequation model, [10], which is in the same spirit as the TSD model. The recent paper of Brio and Hunter, [2], gives a geometrical analysis of equation (2), but does not consider questions which lead to problems like equation (1) and Figure 1; on the other hand, Tabak and Rosales in [16] use an alternative analysis which leads to a different sort of elliptic problem.

The observation we pursue in this paper is that the standard reduction of equation (2) to similarity variables leads to a degenerate elliptic equation 
with a degeneracy which has not previously been studied (but see the recent paper of Choi, Lazer and McKenna, [4], which studies a degeneracy which is almost the same). An examination of the reduction is made elsewhere, [3], but we note here for completeness that the unusual degeneracy in the equation has nothing to do with the fact that (2) is not hyperbolic in time - this is an artifact of the weakly nonlinear geometric optics reduction of the compressible Euler equations; the degeneracy seems to characterize selfsimilar reductions of quasilinear hyperbolic equations in two or more space dimensions.

In this paper, we show the existence of weak solutions for a boundaryvalue problem for equation (1) obtained by making a set of reasonable (but slightly different) assumptions on the boundary values, $g$. We also simplify the shape of the domain in an inessential way. We are now in the process of applying this result to the original Mach reflection model. This involves matching the elliptic to the hyperbolic region via a free-boundary problem, and modifying the elliptic region to be an unbounded domain. However, the degenerate problem we have solved seems to be interesting in its own right, since it is a new type of degeneracy which occurs in a physically interesting problem.

The problem we study in this paper is obtained by letting $x=-\rho$ and $y=\eta$ so that (1) becomes

$$
\begin{aligned}
\left((u+x) u_{x}-\frac{u}{2}\right)_{x}+u_{y y} & =0 \text { in } \Omega \\
u & =g \text { on } \partial \Omega
\end{aligned}
$$

We take $\Omega$ to be a rectangle, $0 \leq x \leq a,-b \leq y \leq b$, in the right half-plane, and the boundary data to satisfy $g=0$ on $x=0$, making this part of the boundary, $\Gamma_{2}$, degenerate. We shall also assume $g \geq 0$ on the rest of the boundary; this, we, shall show, leads to a comparison principle. We shall need more conditions on $g$; see the Assumption in Section 4.1. We look for solutions of ( 3 ) which make the equation elliptic in the interior of $\Omega$.

The degenerate characteristic form at the boundary corresponds to change of type in the flow from hyperbolic (supersonic) to elliptic (subsonic). In fact, the steady TSD equation also has this character, as well as the difficulty that the location in physical space of the sonic line is not known apriori. How the techniques of this paper would apply to the steady TSD equation is an open question. 
Elliptic equations with degenerate characteristic forms have been much studied: [12] surveys the literature up to 1973, while Triebel, [17], extensively covers a number of linear degenerate equations and provides a detailed embedding theory of corresponding weighted Sobolev spaces. However, the prototype degeneracies in [17] apply uniformly to the entire second-order part of the operator. Both [12] and [17] study only linear operators. Linear techniques point to differences between Tricomi type equations (modeled by $x u_{y y}+u_{x x}=0$ ) and Keldysh type equations, [8], (for example $u_{y y}+x u_{x x}=0$ ) in the elliptic region, $x \geq 0$. For the linear Keldysh equation, unlike the Tricomi equation, it is necessary to impose a boundary condition at the degenerate boundary. However, solutions of the Keldysh equation are not differentiable at the degenerate boundary. This was noted by Kohn and Nirenberg, [9]. Because of this behavior, generalizations of Keldysh type equations are not covered by most results on degenerate elliptic equations. Keldysh's equation is closely related to a linearized version of equation (1). Current research in degenerate quasilinear equations focusses on models like the $p$-Laplacian and other geometric problems whose structure appears to be quite different from the problem at hand. Nonlinear equations of Keldysh type have not been studied at all, as far as we know.

The structure of the singularity of equation (3) is discussed in section 2, where we show that solutions exhibit a square root type singularity in the neighborhood of the degenerate boundary. Thus we are led to consider weak solutions of (3) in a Hilbert space with a weighted Sobolev norm.

As a motivation for the choice of the solution space, we study a linear equation associated to the original quasilinear problem. Existence of a weak solution can be proved, as in [12], using the classical Riesz theorem. This is the content of section 3 .

In section 4 we define the weighted Sobolev solution space and the weak formulation for (3), To apply the Browder-Minty theorem, adapting the theory expounded in [13] to a degenerate operator, we need to show that the operator is continuous, pseudo-monotone and coercive. It turns out to be necessary to reformulate the problem so as to obtain a new operator which satisfies all the conditions for the Browder-Minty theorem. We verify the conditions in Section 5. Finally, using the techniques of sub- and super-solutions we show that a solution to the reformulated problem actually satisfies the original equation. 


\section{The Degenerate Boundary and the Com- parison Principle}

In this section, we study equation (3) with Dirichlet boundary conditions on the rectangle $\Omega$. The vanishing of the characteristic form for equation (3) may induce singular behavior in solutions on $\Gamma_{2}$, the part of the boundary on which the equation is degenerate. From the standard theory of elliptic equations we expect the solution to be smooth $\left(C^{2, \mu}\right)$ everywhere in $\Omega$ away from $\Gamma_{2}$. In addition, a comparison principle holds for elliptic solutions of (3), and we formulate this here. The type of singularity expected at the boundary $\Gamma_{2}$ is described first.

\subsection{Singularities in the Solution}

Let us assume that $u \in C^{2}(\Omega) \cap C(\bar{\Omega})$ is a classical solution of equation (3) in $\Omega$, and that it satisfies equation (3) in a weak sense in $\bar{\Omega}$, with $u=0$ on $\Gamma_{2}$. We shall show that if $u$ attains its minimum on $\Gamma_{2}$, then $u$ belongs to $C^{\alpha}(\bar{\Omega}), \alpha>0$, and it must have growth of order $\sqrt{x}$ precisely at $x=0$.

For this purpose, we write a solution $u$ in the form $u(x, y)=x^{\alpha} v(x, y)$, with $\alpha>0$, and $v(0, y) \neq 0$. Then equation (3) yields an equation for $v$ :

$$
\begin{aligned}
& x^{2 \alpha-2}\left\{\alpha(2 \alpha-1) v^{2}+x\left(4 \alpha v v_{x}\right)+x^{2}\left(v_{x}^{2}+v v_{x x}\right)\right\} \\
+ & x^{\alpha-1}\left\{\alpha\left(\alpha-\frac{1}{2}\right) v+x\left(v_{y y}+\left(\frac{1}{2}+2 \alpha\right) v_{x}\right)+x^{2} v_{x x}\right\}=0
\end{aligned}
$$

The relative order of terms depends on whether $\alpha$ is between 0 and $1, \alpha=1$, or $\alpha>1$.

If $0<\alpha<1$, equation (4) implies that at $x=0, \alpha(2 \alpha-1) v^{2}=0$. Since we have assumed that $v(0, y) \neq 0$, it follows that $\alpha=\frac{1}{2}$. Furthermore, in the case when $\alpha=\frac{1}{2}$, equation (4) implies that $v_{x}(0, y)=0$.

If $\alpha=1$, then at $x=0, v\left(v+\frac{1}{2}\right)=0$, so $v(0, y)=-\frac{1}{2}$. Thus $v(x, y)=$ $-\frac{1}{2}+w(x, y)$, where $w(0, y)=0$.

Finally, we consider the case when $\alpha>1$. The leading-order term in (4) is then $\alpha\left(\alpha-\frac{1}{2}\right) x^{\alpha-1} v$. Therefore, at $x=0$, equation (4) implies that $v(0, y)=0$, and so no such solutions exist.

Thus we have proved the following theorem: 
Theorem 1 Let $u \in C^{2}(\Omega) \cap C(\bar{\Omega})$ be a solution of $(1)$ in $\bar{\Omega}$, such that $u=0$ on $\Gamma_{2}$. Then, if $u$ is not identically zero everywhere in $\bar{\Omega}$, either:

(1) $u \in C^{1}(\bar{\Omega})$ and $u$ vanishes exactly to first order at $\Gamma_{2}$, or

(2) $u \in C^{\alpha}(\bar{\Omega})$ for $\alpha \leq \frac{1}{2}$, and $u(x, y)=\sqrt{x} v(x, y)$, where $v(0, y) \neq 0$.

In both cases there are further restrictions on $u$. In case $(1), u_{x}(0, y)=-\frac{1}{2}$; in case (2), then $v_{x}(0, y)=0$.

In particular, if $u \geq 0$ in $\Omega$, then case (1) cannot hold, and we have:

Corollary 1 There is no solution of equation (3) which is $C^{1}$ in $\bar{\Omega}$ and attains its minimum on $\Gamma_{2}$.

If case (2) holds, then $u+x$ is negative near $\Gamma_{2}$ unless $v>0$ on $\Gamma_{2}$. Hence, we have

Corollary 2 A nonnegative solution of (3) which is $C^{\alpha}(\bar{\Omega}), \alpha>0$, and elliptic in $\Omega$ must have growth of order $\sqrt{x}$ precisely at $x=0$ and must satisfy $u>0$ near $\Gamma_{2}$.

\subsection{The Comparison Principle}

We now prove a comparison theorem for the quasilinear equation (3). We prove that every function $v \in C(\bar{\Omega}) \cap C^{2}(\Omega)$, which satisfies the usual conditions for a sub- or super-solution of an elliptic problem, is in fact one, provided in addition it satisfies the condition $v_{x x} \leq 0$ in $\Omega$. This leads to a unique elliptic solution in the class of functions $u$ for which $u_{x x} \leq 0$ in $\Omega$. Moreover, using this comparison principle, we obtain estimates for a weak solution in the neighborhood of the degenerate boundary.

More precisely, let $u \in C(\bar{\Omega}) \cap C^{2}(\Omega)$. We denote by $Q(u)$ the quasilinear operator associated with equation (3),

$$
Q(u) \equiv\left((u+x) u_{x}-\frac{u}{2}\right)_{x}+u_{y y} .
$$

Theorem 2 Let $u, v \in C(\bar{\Omega}) \cap C^{2}(\Omega)$. Suppose that $u$ is a solution of (3) which is elliptic in $\Omega$, and that $v_{x x} \leq 0$ in $\Omega$. If $Q(v) \leq Q(u)=0$ in $\Omega$, and $u \leq v$ on $\partial \Omega$, then $u \leq v$ in $\Omega$. 
Proof: Let $w=u-v$. Then

$$
Q(u)-Q(v)=(u+x) w_{x x}+w_{y y}+\left(u_{x}+v_{x}+\frac{1}{2}\right) w_{x}+v_{x x} w \geq 0 .
$$

Denote by $L$ the linear operator on $w$ defined by (6). Then we have $L w \geq 0$ in $\Omega^{+} \equiv\{(x, y) \in \Omega \mid w(x, y)>0\}$, and $w \leq 0$ on $\partial \Omega$. Since $L$ is elliptic in $\Omega$, with the coefficient $v_{x x}$ of $w$ less than zero, we can apply the maximum principle for linear equations elliptic in the interior of $\Omega$ (see [6, Theorem 3.1, Corollary 3.2]), to see that $w \leq 0$ in $\Omega$, and therefore, $v \geq u$ in $\Omega$. Similarly, one can show that a function $z$, such that $z_{x x} \leq 0, Q(u) \leq Q(z)$ in $\Omega$, and $u \geq z$ on $\partial \Omega$, has the property that $z \leq u$ in $\Omega$.

Corollary 3 If $u, v \in C(\bar{\Omega}) \cap C^{2}(\Omega)$ are two solutions of (3), elliptic in $\Omega$, and $u_{x x}$ and $v_{x x} \leq 0$, then $u \equiv v$ in $\Omega$.

\section{A Linear Model Equation}

As a first step in understanding the behavior of a solution to the Dirichlet problem for the quasilinear equation (3), we study the linear equation obtained by replacing the coefficient $u+x$ of $u_{x x}$ by a fixed function with the singularity we found in the last section. Let $w(x, y)$ be a bounded, measurable solution of (3) which satisfies

$$
w+x \geq 0 \text { in } \bar{\Omega}, \quad w>-x \text { in } \Omega, \quad w=0 \text { on } \Gamma_{2}=\{x=0\} ;
$$

for the calculation below, we choose $w(x, y)=\sqrt{x}$. The linear equation associated with (3) can be written in the form

$$
\left((w+x) u_{x}-\frac{u}{2}\right)_{x}+u_{y y}+c u=c w, \quad c<0 .
$$

Equation (7) is elliptic everywhere in $\bar{\Omega}$ except at the boundary $\Gamma_{2}$, on which the characteristic form vanishes. Appropriate boundary conditions on $\Gamma_{2}$ are found by computing the Fichera function for (7); we summarize the procedure given in [12].

For a linear operator

$$
L(u)=a^{k j}(x) u_{x_{k} x_{j}}+b^{k}(x) u_{x_{k}}+c(x) u,
$$


with non-negative characteristic form

$$
a^{k j}(x) \xi_{k} \xi_{j} \geq 0, \quad \forall \vec{\xi}=\left(\xi_{1}, \xi_{2}\right),
$$

let $\Sigma^{0}$ denote the part of $\partial \Omega$ along which (9) vanishes, and let $\Sigma_{3}=\partial \Omega-\Sigma^{0}$. Definition 1 Let $\vec{n}=\left(n_{1}, n_{2}\right)$ be the inward normal to $\partial \Omega$. The function

$$
b \equiv\left(b^{k}-a_{x_{j}}^{k j}\right) n_{k},
$$

defined on $\Sigma^{0}$, is called the Fichera function.

According to the sign of the Fichera function we divide $\Sigma^{0}$ into three parts, with $\Sigma_{2}$ the part along which $b<0$. As studied in [12], a well-posed boundary value problem for the operator (8) or (7) is the following:

$$
\begin{aligned}
L(u) & =f \text { in } \Omega \\
u & =g \text { on } \Sigma_{2} \cup \Sigma_{3}
\end{aligned}
$$

where $f$ is a given function in $\Omega$, and $g$ a given function on $\Sigma_{2} \cup \Sigma_{3}$.

For equation (7), the Fichera function on $\Gamma_{2}$ has the constant value $-1 / 2$, so that imposing data (for $u$ ) along $\Gamma_{2}$ leads to a well-posed boundary value problem.

\subsection{Existence of a Weak Solution in a Hilbert Space}

In this section we study the existence of a weak solution for the linear degenerate elliptic boundary value problem (7) with

$$
u=g \text { on } \partial \Omega \text {, }
$$

where $w$ is a bounded, measurable function in $\bar{\Omega}$ and $c<0$.

We formulate this problem as a Dirichlet problem with zero boundary data in a standard way: let $v=u-g$, where $g \in C^{2}(\Omega) \cap C(\bar{\Omega})$ is an extension of the boundary data to the whole domain such that $g \geq 0$ in $\bar{\Omega}$ and $g$ never exceeds its maximum on the boundary. Then, existence of a solution of (7) and (10) is equivalent to solving

$$
\begin{aligned}
\left((w+x) v_{x}-\frac{v}{2}\right)+v_{y y}+c v & =f \text { in } \Omega \\
v & =0 \text { on } \partial \Omega .
\end{aligned}
$$


We define a weak solution of problem (11) as follows.

Let $v$ be a function in $C_{0}^{2}(\bar{\Omega})=\left\{v \in C^{2}(\bar{\Omega}) \mid v=0\right.$ on $\left.\partial \Omega\right\}$, and $z$ be in $C_{0}^{1}(\bar{\Omega})$. Multiplying the PDE in (11) by a test function $z \in C_{0}^{1}$ and integrating by parts we obtain

$$
-\int_{\Omega}\left((w+x) v_{x}-\frac{v}{2}\right) z_{x}+v_{y} z_{y}-c v z=\int_{\Omega} f u, \quad \forall z \in C_{0}^{1}(\bar{\Omega}) .
$$

For functions in $C_{0}^{1}(\bar{\Omega})$ we introduce the scalar product

$$
(v, z)_{\mathcal{H}} \equiv \int_{\Omega}(w+x) v_{x} z_{x}+v_{y} z_{y}+v z .
$$

Define $\mathcal{H}$ to be the completion of $C_{0}^{1}(\bar{\Omega})$ in the norm defined by this scalar product.

For $v, z \in C_{0}^{1}$ we study the bilinear form

$$
B(v, z) \equiv-\int_{\Omega}\left((w+x) v_{x}-\frac{v}{2}\right) z_{x}+v_{y} z_{y}-c v z .
$$

Proposition 1 For each fixed $z \in C_{0}^{1}$, the operator $v \mapsto B(v, z)$ is a linear, bounded functional on $v \in \mathcal{H}$.

Proof: It is enough to show that for all $v, z \in C_{0}^{1}, B(v, z)$ satisfies the inequality

$$
|B(v, z)| \leq M \sqrt{\int_{\Omega} z_{z}^{2}+z_{y}^{2}+z^{2}}\|v\|_{\mathcal{H}},
$$

where $M$ depends only on the coefficients of the operator $B$. Inequality (12) follows from the Cauchy-Schwartz inequality in $\left(L^{2}\right)^{3}$. That is,

$$
\begin{aligned}
|B(v, z)| & =\left|\int_{\Omega}\left((w+x) v_{x}-\frac{v}{2}\right) z_{x}+v_{y} z_{y}-c v z\right| \\
& \leq\left(\int_{\Omega}\left((w+x) v_{x}-\frac{v}{2}\right)^{2}+v_{y}^{2}+c^{2} v^{2}\right)^{\frac{1}{2}}\left(\int_{\Omega} z_{x}^{2}+z_{y}^{2}+z^{2}\right)^{\frac{1}{2}} \\
& =M \sqrt{\int_{\Omega} z_{x}^{2}+z_{y}^{2}+z^{2}}\|v\|_{\mathcal{H}}
\end{aligned}
$$

where $M=\max \left\{\sqrt{(w+x)}, 1,|c|+\frac{1}{2}\right\}$. From this inequality we conclude that $B(v, z)$ can be extended to $v \in \mathcal{H}$, and that for each fixed $z \in C_{0}^{1}, B(v, z)$ is a bounded, linear functional on $\mathcal{H}$. 
Definition 2 Let $f \in L^{2}(\Omega)$. A function $v \in \mathcal{H}$ is called a weak solution of (11) if for all $u \in C_{0}^{1}$ the following equation holds:

$$
\int_{\Omega} f u=B(v, u)
$$

Theorem 3 Let $f \in L^{2}(\Omega)$ and $c<0$. Then there exists a weak solution $v \in \mathcal{H}$ of the problem (11).

The existence of a weak solution of (11) in the Hilbert space $\mathcal{H}$ is a consequence of a more general result proved by Oleĭnik and Radkevič [12, Theorem 1.4.1, page 29]. Note that these authors require more smoothness in $w$ than we have; but it is used only to straighten the degenerate part of the boundary. For the problem we consider here, we do not need derivatives of $w$ at $\Gamma_{2}$.

\section{The Weighted Sobolev Space Setting}

In this section we formulate the problem of finding a weak solution of the Dirichlet problem for equation (3) in a weighted Sobolev space defined below. The extension from the linear case is nontrivial because of the lack of smoothness of $u$ at $\Gamma_{2}$. We get around this difficulty by defining a new operator with a fixed singularity at $\Gamma_{2}$. In Section 5 we show that the new operator is continuous, coercive, and pseudo-monotone, and use the Browder-Minty theorem to show the existence of a weak solution. Then, using the techniques of sub-and super-solutions, we show that a solution to the redefined problem actually satisfies the original problem (3).

\subsection{Modification of the Problem}

We now make an assumption on the boundary data $g$ which is necessary for redefining the operator as outlined above.

Assumption: The boundary data $g$ can be extended to a $C^{2}(\Omega)$ function everywhere in $\Omega$ with the properties $g \geq 0, g_{x x}<0$ and $\epsilon \sqrt{x} \leq g \leq(1 / \epsilon) \sqrt{x}$ in $\Omega$, for some $\epsilon>0$. The maximum of $g$ is attained on $\partial \Omega$, and we can write $g(x, y)=\sqrt{x} \bar{g}(x, y)$, where $\bar{g}$ is smooth. 
Note that this is stronger than the conditions in problem (1), and is inconsistent with the behavior of $g$ on $\Gamma_{\infty}$ in Figure 1. We shall consider modifications in future work.

To use the monotone operator framework, we recast the problem as a Dirichlet problem with zero boundary data, by the substitution $u=v+g$. We obtain in place of $(3)$

$$
\begin{array}{rlrl}
-\left((v+g+x) v_{x}+\left(g_{x}-\frac{1}{2}\right) v\right)_{x}-v_{y y} & =f & \text { in } \Omega \\
v & =0 & & \text { on } \partial \Omega .
\end{array}
$$

Here

$$
f=g_{y y}+\left((g+x) g_{x}\right)_{x}-\frac{1}{2} g_{x} .
$$

The weak formulation of (14) is as follows. Let $v \in C^{2}(\Omega)$ satisfy (14) and let $w$ be in $C_{0}^{1}(\bar{\Omega})$. Multiplying the PDE in (14) by the test function $w$ and integrating by parts one obtains

$$
B(v, w) \equiv \int_{\Omega}\left((v+g+x) v_{x}+\left(g_{x}-\frac{1}{2}\right) v\right) w_{x}+v_{y} w_{y}=\int_{\Omega} f w .
$$

For functions $v, w$ in $C_{0}^{1}(\bar{\Omega})$ we introduce the scalar product

$$
(v, w)_{\mathcal{H}_{\alpha}} \equiv \int_{\Omega} x^{\alpha} v_{x} w_{x}+v_{y} w_{y}+v w
$$

Define the Hilbert space $\mathcal{H}_{\alpha}$ to be the completion of $C_{0}^{1}(\bar{\Omega})$ in the norm defined by (17). We have a form of the Poincaré inequality which makes this norm equivalent to :

$$
\|u\|^{2} \equiv \int_{\Omega} x^{\alpha} v_{x}^{2}+v_{y}^{2}
$$

Proposition 2 Let $0<\alpha<2$. There exists a constant $C>0$ such that for every $v \in \mathcal{H}_{\alpha}$ the following inequality holds:

$$
\int_{\Omega} v^{2} d x d y \leq C \int_{\Omega} x^{\alpha} v_{x}^{2} d x d y
$$


Proof: We may assume $v \in C_{0}^{1}(\bar{\Omega})$. An integration by parts and the CauchySchwartz inequality imply

$$
\begin{aligned}
\int_{\Omega} v^{2} d x d y & =-\int_{\Omega} x \frac{\partial v^{2}}{\partial x} d x d y \\
& =-2 \int_{\Omega} x^{1-\alpha / 2} v x^{\alpha / 2} \frac{\partial v}{\partial x} d x d y \\
& \leq 2 \max _{\Omega} x^{1-\alpha / 2}\left(\int_{\Omega} v^{2}\right)^{\frac{1}{2}}\left(\int_{\Omega} x^{\alpha} v_{x}^{2}\right)^{\frac{1}{2}}
\end{aligned}
$$

Therefore,

$$
\int_{\Omega} v^{2} d x d y \leq C \int_{\Omega} x^{\alpha} v_{x}^{2} d x d y,
$$

where $C=\left(2 \max _{\Omega} x^{1-\alpha / 2}\right)^{2}$.

As a consequence, we have:

Proposition 3 The norms defined by (18) and (17) are equivalent.

For the remainder of this paper we shall use (18) for the norm, and will modify (17) appropriately for the corresponding scalar product.

Definition 3 Let $f \in L^{2}(\Omega)$. A function $v \in \mathcal{H}_{\alpha}$ is called a weak solution of (14) if (16) holds for every $w \in C_{0}^{1}(\bar{\Omega})$.

We anticipate a couple of difficulties in proving existence of a weak solution using (16). First, if $u$ is not greater than $-x$ in $\Omega$, the equation will no longer be elliptic. As we shall see, the value of $\alpha$ for which we get an existence theorem is $\alpha=\frac{1}{2}$; however for no value of $\alpha$ is (16) defined for all $v$, $w$ in $\mathcal{H}_{\alpha}$, continuous on $\mathcal{H}_{\alpha} \times \mathcal{H}_{\alpha}$ and coercive, even for $v \geq-g$. Assuming that we are looking for an elliptic solution $u$ of equation (3), then, from the comparison principle of Theorem 2, we expect $u$ to be squeezed between suband super-solutions given by $\delta \sqrt{x}, M \sqrt{x}$, respectively, for any $\delta \leq \epsilon$ and $M \geq(1 / \epsilon)$. This is equivalent to saying that $v+g$ in problem (14) exhibits square-root behavior on $\Gamma_{2}$. Therefore, we redefine $B(v, w)$ by introducing a cut-off function for the coefficient $v+g$ in such a way that the modified coefficient exhibits square-root behavior on $\Gamma_{2}$. We define the new operator $B_{g}(v, w)$ as follows. 
Let $\epsilon>0$ be the constant which bounds $g$ in the Assumption. We define

$$
\bar{u}= \begin{cases}v+g & \text { if } \epsilon \sqrt{x} \leq v+g \leq(1 / \epsilon) \sqrt{x} \\ \epsilon \sqrt{x} & \text { if } v+g<\epsilon \sqrt{x} \\ (1 / \epsilon) \sqrt{x} & \text { if }(1 / \epsilon) \sqrt{x}<v+g\end{cases}
$$

Now define

$$
B_{g}(v, w)=\int_{\Omega}\left((\tilde{u}+x) v_{x}+\left(g_{x}-\frac{1}{2}\right) v\right) w_{x}+v_{y} w_{y} .
$$

We shall prove the existence of a solution in $\mathcal{H}_{\alpha}, \alpha=\frac{1}{2}$, for

$$
B_{g}(v, w)=\int_{\Omega} f w .
$$

Then we shall show that the solution we have found satisfies $\epsilon \sqrt{x} \leq v+g \leq$ $(1 / \epsilon) \sqrt{x}$. Therefore, $B_{g}(v, w)$ coincides with $B(v, w)$ for this solution, and $v$ satisfies the original problem.

We have introduced a new difficulty: $B_{g}$ depends on $g$, as does $f$ in (21). We handle this by supposing $f$ to be an arbitrary function, in (21), (not related to $g$ by (15)), and then letting $f$ be the function defined by (15) to obtain the existence of a weak solution for problem (14).

Notice that for each fixed $v \in \mathcal{H}_{\alpha}, B_{g}(v, w)$ is a linear functional in $w \in C_{0}^{1}$. We show that $w \mapsto B_{g}(v, w)$ is bounded and can be extended to a bounded, linear functional on $\mathcal{H}_{\alpha}$, for $\alpha=\frac{1}{2}$. In order to do this, we first investigate some properties of the space $\mathcal{H}_{\alpha}$.

\subsection{Embeddings of the Hilbert Space $\mathcal{H}_{\alpha}$}

Let $(\Omega, \mathcal{B}, \mu)$ be the measure space defined on $\Omega$ with product measure $\mu$ given by

$$
d \mu=\frac{1}{x^{\beta}} d x d y .
$$

The value of $\beta$ will be specified later. We denote by $L^{2}(\Omega, \beta)$ the space

$$
L^{2}(\Omega, \beta) \equiv\left\{u \mid u \text { measurable and } \int_{\Omega}|u|^{2} d \mu<\infty\right\} .
$$

Clearly, $L^{2}(\Omega, \beta)$ is a Hilbert space with scalar product

$$
(u, v)_{L^{2}(\Omega, \beta)} \equiv \int_{\Omega} u v d \mu=\int_{\Omega} u v \frac{d x}{x^{\beta}} d y .
$$


Theorem 4 The space $\mathcal{H}_{\alpha}$, for each $0<\alpha<1$, is continuously embedded in $L^{2}(\Omega, \beta)$, for any $\beta \leq 2-\alpha$.

Proof: Let $u \in C_{0}^{1}(\bar{\Omega})$. If $\beta \neq 1$, an integration by parts and the CauchySchwartz inequality yield

$$
\begin{aligned}
\|u\|_{L^{2}(\Omega, \beta)}^{2} & =\int_{\Omega} \frac{u^{2}}{x^{\beta}} d x d y \\
& =-\frac{1}{1-\beta} \int_{\Omega} x^{1-\beta} \frac{\partial u^{2}}{\partial x} d x d y \\
& =-\frac{2}{1-\beta} \int_{\Omega} x^{1-\beta} u \frac{\partial u}{\partial x} \\
& =-\frac{2}{1-\beta} \int_{\Omega} x^{\delta} \frac{u}{x^{\beta / 2}} x^{1-\beta / 2-\delta} \frac{\partial u}{\partial x} d x d y \\
& \leq \frac{2}{|1-\beta|} \max _{\Omega} x^{\delta}\left(\int_{\Omega} \frac{u^{2}}{x^{\beta}} d x d y\right)^{\frac{1}{2}}\left(\int_{\Omega} x^{2-\beta-2 \delta} u_{x}^{2}\right)^{\frac{1}{2}} \\
& \leq C\|u\|_{L^{2}(\Omega, \beta)}\|u\|_{\mathcal{H}_{\alpha}}
\end{aligned}
$$

where $\delta \geq 0$, and $\alpha=2-\beta-2 \delta$. Therefore, if $\beta \leq 2-\alpha$, there exists a constant $C \equiv \frac{2}{|1-\beta|} \max _{\Omega} x^{1-(\alpha+\beta) / 2}$ such that

$$
\|u\|_{L^{2}(\Omega, \beta)} \leq C\|u\|_{\mathcal{H}_{\alpha}} .
$$

If $\beta=1$, integration by parts introduces a logarithmic factor, and the rest of the argument is the same. Since $C_{0}^{1}(\bar{\Omega})$ is dense in $\mathcal{H}_{\alpha}$, inequality (22) holds for all functions $u$ in $\mathcal{H}_{\alpha}$.

For future purposes, we shall need the fact that the embeddings are actually compact. The following theorem is proved in the Appendix.

Theorem 5 The space $\mathcal{H}_{\alpha}, 0<\alpha<1$, is compactly embedded in $L^{2}(\Omega, \beta)$ for all $\beta<2-\alpha$. 


\subsection{Weak Formulation of the Modified Problem}

We now return to the problem of section 4.1. As described at the end of that section, we replace (14) by

$$
\begin{aligned}
-\left((\tilde{u}+x) v_{x}+\left(g_{x}-\frac{1}{2}\right) v\right)_{x}-v_{y y} & =f, \text { in } \Omega \\
v & =0, \text { on } \partial \Omega
\end{aligned}
$$

where $f$ is now not considered to depend on $g$. Equation (20) defines the bivariate form associated with the weak formulation of this problem.

In this section we show that $B_{g}$ is bounded on $\mathcal{H}_{\frac{1}{2}} \times \mathcal{H}_{\frac{1}{2}}$, then we define a nonlinear mapping $T: \mathcal{H}_{\frac{1}{2}} \rightarrow \mathcal{H}_{\frac{1}{2}}^{*}$ associated with $B_{g}(T$ also depends on $g$ ). In Section 5, we establish that this mapping is continuous, pseudo-monotone and coercive, and then use a version of the Browder-Minty theorem to obtain the existence of a weak solution to $(21)$ in $\mathcal{H}_{\frac{1}{2}}$.

To define $T$ we first prove

Theorem 6 For each $v \in \mathcal{H}_{\frac{1}{2}}(\Omega)$, and $w \in C_{0}^{1}(\Omega)$, the following inequality holds:

$$
\left|B_{g}(v, w)\right| \leq \tilde{C}\|v\|_{\mathcal{H}_{\frac{1}{2}}}\|w\|_{\mathcal{H}_{\frac{1}{2}}}
$$

where $\widetilde{C}$ depends on $g$.

Proof: We apply the Cauchy-Schwartz inequality to $B_{g}(v, w)$ and use the embedding of $\mathcal{H}_{\frac{1}{2}}$ in $L^{2}(\Omega, \beta)$ with $\beta=3 / 2$ in the following way:

$$
\begin{array}{r}
\quad\left|B_{g}(v, w)\right|=\left|\int_{\Omega} \sqrt{\tilde{u}+x} v_{x} \sqrt{\tilde{u}+x} w_{x}+\left(g_{x}-\frac{1}{2}\right) \frac{v}{x^{1 / 4}} x^{1 / 4} w_{x}+v_{y} w_{y}\right| \\
\leq\left(\int_{\Omega}(\tilde{u}+x) v_{x}^{2}+\left(g_{x}-\frac{1}{2}\right)^{2} \frac{v^{2}}{\sqrt{x}}+v_{y}^{2}\right)^{\frac{1}{2}}\left(\int_{\Omega}(\tilde{u}+x) w_{x}^{2}+\sqrt{x} w_{x}^{2}+w_{y}^{2}\right)^{\frac{1}{2}}
\end{array}
$$

We estimate each integral in the first factor of the product separately. To estimate the second integral we use the assumption that there exists a smooth function $\bar{g}$ with $g(x, y)=\sqrt{x} \bar{g}(x, y)$ and $\epsilon \leq \bar{g}(x, y) \leq 1 / \epsilon$. Using this property of $g$ we obtain

$$
\left|g_{x}\right| \leq C x^{-1 / 2}+D
$$


Therefore, we have

$$
\int_{\Omega}\left(g_{x}-\frac{1}{2}\right)^{2} \frac{v^{2}}{\sqrt{x}} \leq C^{2} \int_{\Omega} \frac{v^{2}}{x^{3 / 2}}+E \int_{\Omega} \frac{v^{2}}{x}+F \int_{\Omega} \frac{v^{2}}{\sqrt{x}}
$$

Now, from the embedding of $\mathcal{H}_{\frac{1}{2}}$ in $L^{2}(\Omega, \beta)$, for $\beta \leq 3 / 2$, we obtain

$$
\int_{\Omega}\left(g_{x}-\frac{1}{2}\right)^{2} \frac{v^{2}}{\sqrt{x}} \leq K\|v\|_{\mathcal{H}_{\frac{1}{2}}}^{2}
$$

where $K$ depends on $g$.

We estimate the first integral in both factors of (25) using the bound on $\bar{u}:$

$$
\begin{aligned}
\int_{\Omega}(\tilde{u}+x) v_{x}^{2} & \leq \int_{\Omega}((1 / \epsilon) \sqrt{x}+x) v_{x}^{2} \\
& \leq(1 / \epsilon) \max _{\Omega}\{1+\epsilon \sqrt{x}\} \int_{\Omega} \sqrt{x} v_{x}^{2} \\
& \leq C_{1}(\epsilon)\|v\|_{\mathcal{H}_{\frac{1}{2}}}^{2}
\end{aligned}
$$

The second factor of (25) is then bounded:

$$
\int_{\Omega}(\tilde{u}+x) w_{x}^{2}+\sqrt{x} w_{z}^{2}+w_{y}^{2} \leq C_{2}(\epsilon)\|w\|_{\mathcal{H}_{\frac{1}{2}}}^{2} .
$$

Combining the last three estimates we see that there is a constant $\tilde{C}(g)>0$ such that (24) holds.

Corollary 4 The operator $w \mapsto B_{g}(v, w)$, defined on $w \in C_{0}^{1}(\bar{\Omega})$, is a bounded, linear functional for each fixed $v \in \mathcal{H}_{\frac{1}{2}}(\Omega)$. Moreover, it can be extended to a bounded, linear functional on $\mathcal{H}_{\frac{1}{2}}(\Omega)$.

Corollary 5 There exists a mapping

$$
\mathcal{H}_{\frac{1}{2}} \ni v \mapsto T(v) \in \mathcal{H}_{\frac{1}{2}}^{*}
$$

such that

$$
B_{g}(v, w)=\langle T(v), w\rangle
$$

for all $v$ in $\mathcal{H}_{\frac{1}{2}}, w \in \mathcal{H}_{\frac{1}{2}}$. Furthermore, the nonlinear mapping is bounded. 
Remark The space $\mathcal{H}_{\frac{1}{2}}^{*}$ is the negative norm weighted Sobolev space dual to $\mathcal{H}_{\frac{1}{2}}$ under the pairing

$$
\langle v, w\rangle=\int_{\Omega} v w d x d y .
$$

A weak solution of (21) is a function $v \in \mathcal{H}_{\frac{1}{2}}$ such that

$$
B_{g}(v, w)=\langle T(v), w\rangle=\langle f, w\rangle, \quad \forall w \in \mathcal{H}_{\frac{1}{2}} .
$$

To prove existence of a solution, we use a version of the classical BrowderMinty theorem (Theorem 7, below) which gives the existence of a weak solution if $T$ is continuous, coercive, and pseudo-monotone.

We recall some definitions from [13].

Definition 4 Let $X$ be a real, reflexive Banach space. We say that the mapping $T: X \rightarrow X^{*}$ is coercive if

$$
\frac{\langle T(u), u\rangle}{\|u\|} \rightarrow \infty \text { as }\|u\| \rightarrow \infty .
$$

Definition 5 Let $X$ be a real, reflexive Banach space. An operator $T: X \rightarrow$ $X^{*}$ is called pseudo-monotone if $T$ is bounded and if whenever

$$
u_{n} \rightarrow u \text { in } X
$$

and

$$
\limsup _{n \rightarrow \infty}\left\langle T\left(u_{n}\right), u_{n}-u\right\rangle \leq 0
$$

it follows that

$$
\liminf _{n \rightarrow \infty}\left\langle T\left(u_{n}\right), u_{n}-v\right\rangle \geq\langle T(u), u-v\rangle \quad \forall v \in X .
$$

Theorem 7 (The Browder-Minty Theorem; [13, Theorem 9.57, page 367]) Let $X$ be a real, reflexive Banach space and suppose that $T: X \rightarrow X^{*}$ is continuous, coercive and pseudo-monotone. Then for every $f \in X^{*}$ there exists a solution $u \in X$ of the equation

$$
T(u)=f .
$$

In the next section we prove that the operator $T$ defined in Corollary 5 of Theorem 6 is continuous, pseudo-monotone and coercive. 


\section{Existence of a Weak Solution}

In this section we verify the properties of $T$, apply Theorem 7 , and then show that the solution solves the original problem.

\subsection{Properties of $T$}

We show first that $T$ is continuous by showing that each coefficient of $T$, as a function of $\left(x^{-3 / 4} u(x, y), x^{1 / 4} u_{x}(x, y), u_{y}(x, y)\right) \in\left(L^{2}\right)^{3}$, is continuous and bounded, and can be considered as a Nemytskii operator from $\left(L^{2}\right)^{3}$ to its dual. The continuity of Nemytskii operators (see [11], [13]), then implies the continuity of $T$. The following argument is adapted from [11] and [13] to the weighted Sobolev space we are dealing with here. We recall the definition of a Nemytskii operator.

Definition 6 For $(\xi, \omega) \in \Omega \times \mathbf{R}^{m}$, we say that a function $f(\xi, \omega)$ satisfies the Caratheodory conditions if the mapping $\omega \mapsto f(\xi, \omega)$ is continuous for almost every $\xi \in \Omega$, and $\xi \mapsto f(\xi, \omega)$ is measurable for every $\omega \in \mathbf{R}^{m}$. For such an $f$ and any function $\omega: \Omega \rightarrow \mathbf{R}^{m}$,

$$
a(\omega)(\xi) \equiv f(\xi, \omega(\xi))
$$

is called a Nemytskii operator.

Theorem 8 ([11],[13]) Let

$$
\Omega \times \mathbf{R}^{m} \ni(\xi, \omega) \mapsto f(\xi, \omega) \in \mathbf{R}^{m}
$$

satisfy the Caratheodory conditions, and

$$
|f(\xi, \omega)| \leq C|\omega|^{p-1}+h(\xi)
$$

where $p \in(1, \infty), h \in L^{q},\left(\frac{1}{p}+\frac{1}{q}=1\right)$. Then the Nemytskii operator defined by (27) is a bounded and continuous map from $L^{p}$ to $L^{q}$.

We now apply the calculus of Nemytskii operators to prove the continuity of $T$.

Theorem 9 The operator $T$ is continuous. 
Proof: To study the coefficients of $T$ we write $\langle T(v), w\rangle$ as

$$
\begin{aligned}
\langle T(v), w\rangle & =\int_{\Omega} \frac{(\tilde{u}+x) x^{1 / 4} v_{x}}{\sqrt{x}} x^{1 / 4} w_{x} \\
& +\int_{\Omega}\left(g_{x}-\frac{1}{2}\right) \frac{v}{x^{3 / 4}} \sqrt{x} x^{1 / 4} w_{x}+\int_{\Omega} v_{y} w_{y} .
\end{aligned}
$$

Since $v \in \mathcal{H}_{\frac{1}{2}}$ implies $v \in L^{2}(\Omega, \beta)$ with $\beta=3 / 2$, we know that $v /\left(x^{3 / 4}\right) \in L^{2}$. Therefore, we may consider the coefficients of $T$ as functions from $\left(L^{2}\right)^{3}$ to $L^{2}$ by defining the symbol vector

$$
\omega=\left(\omega_{1}, \omega_{2}, \omega_{3}\right)=\left(\frac{v}{x^{3 / 4}}, x^{1 / 4} v_{x}, v_{y}\right)
$$

Then the coefficients of the symbol of $T$ become

$$
\begin{aligned}
& a_{1}(x, y, \omega)=\frac{\left(\widetilde{\omega_{1}}+x\right) \omega_{2}}{\sqrt{x}} \\
& a_{2}(x, y, \omega)=\left(g_{x}-\frac{1}{2}\right) \sqrt{x} \omega_{1} \\
& a_{3}(x, y, \omega)=\omega_{3} .
\end{aligned}
$$

Here, $\widetilde{\omega_{1}}$ denotes the function $\widetilde{\omega_{1}} \equiv \omega_{1} x^{3 / 4}+g$, which satisfies the bounds

$$
\epsilon \sqrt{x} \leq \widetilde{\omega_{1}} \leq(1 / \epsilon) \sqrt{x} .
$$

Now, all three functions $a_{1}, a_{2}$, and $a_{3}$ are continuous for almost every $(x, y) \in$ $\Omega$, and measurable for every $\omega \in \mathbf{R}^{3}$. Moreover,

$$
\begin{aligned}
& \left|a_{1}(x, y, \omega)\right| \leq \frac{(1 / \epsilon) \sqrt{x}+x}{\sqrt{x}}\left|\omega_{2}\right| \leq C_{1}\left|\omega_{2}\right| \\
& \left|a_{2}(x, y, \omega)\right| \leq\left|g_{x}-\frac{1}{2}\right| \sqrt{x}\left|\omega_{1}\right| \leq\left(\frac{K}{\sqrt{x}}+D\right) \sqrt{x}\left|\omega_{1}\right| \leq C_{2}\left|\omega_{1}\right| \\
& \left|a_{3}(x, y, \omega)\right|=\left|\omega_{3}\right| .
\end{aligned}
$$

This shows that the conditions of Theorem 8 hold with $p=2$, and thus the symbol is continuous on a weighted $L^{2}$ space and the corresponding map $T$ is continuous from $\mathcal{H}_{\frac{1}{2}}$ to $\mathcal{H}_{\frac{1}{2}}^{*}$. 
To verify pseudo-monotonicity we use the approach presented in [13]; we show that the operator $T$ is of calculus of variations type, which implies that $T$ is pseudo-monotone. We state the definition and theorem from [13, pages 367-368].

Definition 7 An operator $T: X \rightarrow X^{*}$ is said to be of calculus of variations type if it is bounded, and it has the representation $T(v)=\hat{T}(v, v)$ where the mapping from $X \times X$ to $X^{*}$ given by $(v, z) \mapsto \hat{T}(v, z)$ satisfies the following properties:

1. The mappings $z \mapsto \hat{T}(v, z)$ and $v \mapsto \hat{T}(v, z)$ are bounded and continuous.

2. For each $v \in X$,

$$
\langle\hat{T}(v, v)-\hat{T}(v, z), v-z\rangle \geq 0, \quad \forall z \in X .
$$

3. If $v_{n} \rightarrow v$ in $X$, and $\left\langle\hat{T}\left(v_{n}, v_{n}\right)-\hat{T}\left(v_{n}, v\right), v_{n}-v\right\rangle \rightarrow 0$, then for every $z \in X$

$$
\hat{T}\left(v_{n}, z\right) \rightarrow \hat{T}(v, z) \quad \text { in } X^{*} \text {. }
$$

4. If $v_{n} \rightarrow v$ in $X$, and $\hat{T}\left(v_{n}, z\right) \rightarrow \psi$ in $X^{*}$, then

$$
\left\langle\hat{T}\left(v_{n}, z\right), v_{n}\right\rangle \rightarrow\langle\psi, v\rangle .
$$

Theorem 10 ([13]) If $T$ is of calculus of variations type, then $T$ is pseudomonotone.

To show that $T$ is of calculus of variations type, we define $\bar{B}_{g}(v, z, w)$ by

$$
\bar{B}_{g}(v, z, w) \equiv \int_{\Omega}\left((\tilde{u}+x) z_{x}+\left(g_{x}-\frac{1}{2}\right) z\right) w_{x}+z_{y} w_{y} .
$$

Using the same argument as in Theorem 6 , we can show that there is a mapping $(v, z) \mapsto \hat{T}(v, z)$, from $\mathcal{H}_{\frac{1}{2}} \times \mathcal{H}_{\frac{1}{2}}$ to $\mathcal{H}_{\frac{1}{2}}^{*}$, such that

$$
\bar{B}_{g}(v, z, w)=\langle\hat{T}(v, z), w\rangle \quad \text { for all } w \in \mathcal{H}_{\frac{1}{2}},
$$

and $T(v)=\hat{T}(v, v)$. Note that $\bar{B}$ is linear in its second and third arguments, while its dependence on $v$ is bounded (by the cut-off in $\tilde{u}$ ) and does not involve derivatives. Thus it is straightforward to verify the conditions of Definition 7 , as we now do.

We need the following proposition. 
Proposition 4 If $v_{n} \rightarrow v$ in $L^{2}(\Omega, \beta)$, then $\tilde{u}_{n} \rightarrow \tilde{u}$ in $L^{2}(\Omega, \beta)$, where $\tilde{u}_{n}$ and $\tilde{u}$ are defined as in (19).

Proof: Let $\Omega^{\epsilon}=\{x \in \Omega \mid v+g<\epsilon \sqrt{x}\}, \Omega^{1 / \epsilon}=\{x \in \Omega \mid v+g>(1 / \epsilon) \sqrt{x}\}$, and let $\Omega^{v+g}$ denote the subset of $\Omega$ on which $\tilde{u}=v+g$. Similarly, define the sets $\Omega_{n}^{\epsilon}, \Omega_{n}^{1 / \epsilon}$, and $\Omega^{v_{n}+g}$, corresponding to $v_{n}$. Now, we write the $L^{2}(\Omega, \beta)$ norm of $\tilde{u}_{n}-\tilde{u}$ as follows:

$$
\begin{aligned}
\left\|\tilde{u}_{n}-\tilde{u}\right\|^{2} & =\int_{\Omega_{n}^{\varsigma} \cap \Omega^{夭}}\left(\tilde{u}_{n}-\tilde{u}\right)^{2} d \mu+\int_{\Omega_{n}^{1 / \epsilon} \cap \Omega^{1 / \epsilon}}\left(\tilde{u}_{n}-\tilde{u}\right)^{2} d \mu \\
& +\int_{\Omega_{\mathbf{v}_{n}+g_{\cap} \cap \Omega^{v+g}}}\left(\tilde{u}_{n}-\tilde{u}\right)^{2} d \mu+\int_{\Omega^{c}}\left(\tilde{u}_{n}-\tilde{u}\right)^{2} d \mu,
\end{aligned}
$$

where $\Omega^{C}$ denotes the subset of $\Omega$ on which $\tilde{u}_{n}$ and $\tilde{u}$ are defined using different formulas in definition (19). Since $v_{n} \rightarrow v$ in $L^{2}(\Omega, \beta)$, the measure of the set $\Omega^{C}$ tends to 0 . Also, since $\tilde{u}_{n}$ and $\tilde{u}$ are bounded on $\Omega^{C}$ the integral tends to zero, that is,

$$
\int_{\Omega^{o}}\left(\tilde{u}_{n}-\tilde{u}\right)^{2} d \mu \leq \int_{\Omega^{o}}\left(\left|\tilde{u}_{n}\right|+|\tilde{u}|\right)^{2} d \mu \leq K \mu\left\{\Omega^{C}\right\} \rightarrow 0 .
$$

Now, since $v_{n} \rightarrow v$ in $L^{2}(\Omega, \beta)$, we have

$$
\int_{\Omega^{v_{n}+g} \cap \Omega^{v+g}}\left(\tilde{u}_{n}-\tilde{u}\right)^{2} d \mu=\int_{\Omega^{v_{n}+g} \cap \Omega^{v+g}}\left(v_{n}-v\right)^{2} d \mu \rightarrow 0 .
$$

Finally, since the integrands in the remaining integrals are identically 0 , we see that $\left\|\tilde{u}_{n}-\tilde{u}\right\| \rightarrow \hat{0}$.

Now we can prove

Theorem 11 The operator $T$ is of calculus of variations type.

Proof: Boundedness of $T$ follows from Theorem 6; we write $T(v)=\hat{T}(v, v)$, where $\hat{T}(v, z)$ is given by $(29)$. We show that the operator $\hat{T}(v, z)$ satisfies the conditions of Definition 7:

1. Boundedness of the mapping $z \mapsto \hat{T}(v, z)$ follows in the same way as the boundedness of $B(v, w)$, proved in Theorem 6. Continuity follows since $z \mapsto$ $\hat{T}(v, z)$ is linear. Boundedness of the mapping $v \mapsto \hat{T}(v, z)$ also follows from Theorem 6 . Using the same arguments as in Theorem 9 to prove continuity of $T$, we can show that $v \mapsto \hat{T}(v, z)$ is a Nemytskii operator, and, therefore, 
is continuous.

2. Now we prove that inequality (28) holds. Recall that we have extended the boundary data $g$ to a function on $\Omega$ with the property that $g_{x x} \leq 0$. Therefore, we have

$$
\begin{aligned}
& \langle\hat{T}(v, v)-\hat{T}(v, z), v-z\rangle \\
= & \int_{\Omega}(\tilde{u}+x)\left(v_{x}-z_{x}\right)^{2}+\left(g_{x}-\frac{1}{2}\right)(v-z)(v-z)_{x}+\left(v_{y}-z_{y}\right)^{2}
\end{aligned}
$$

We estimate the middle term in (30) by integrating by parts and taking into account that $v$ and $z$ vanish on $\partial \Omega$, to obtain

$$
\int_{\Omega}\left(g_{z}-\frac{1}{2}\right)(v-z)(v-z)_{x}=-\int_{\Omega} \frac{1}{2} g_{x z}(v-z)^{2} \geq 0
$$

Since $v-z \in L^{2}(\Omega, \beta)$ for $\beta=3 / 2$, and $g_{x x} \leq 0$, this integral is bounded and non-negative. Therefore, (28) follows from (30) and (31).

3. Let $v_{n} \rightarrow v$ in $\mathcal{H}_{\frac{1}{2}}$. By the compactness of the embedding of $\mathcal{H}_{\frac{1}{2}}(\Omega)$ in $L^{2}(\Omega, \beta)$, for $\beta<3 / 2$, we have $\tilde{u}_{n} \rightarrow \tilde{u}$ (strongly) in $L^{2}(\Omega, \beta)$. Therefore, even without the hypothesis $\left\langle\hat{T}\left(v_{n}, v_{n}\right)-\hat{T}\left(v_{n}, v\right), v_{n}-v\right\rangle \rightarrow 0$ we obtain $\bar{B}_{g}\left(v_{n}, z, w\right) \rightarrow \bar{B}(v, z, w)$, or equivalently,

$$
\hat{T}\left(v_{n}, z\right) \rightarrow \hat{T}(v, z) \text { in } \mathcal{H}_{\frac{1}{2}}^{*} .
$$

4. Let $v_{n} \rightarrow v$ in $\mathcal{H}_{\frac{1}{2}}$, and $\hat{T}\left(v_{n}, z\right) \rightarrow \psi$ in $\mathcal{H}_{\frac{1}{2}}^{*}$. We need to show that

$$
\left\langle\hat{T}\left(v_{n}, z\right), v_{n}\right\rangle=\left\langle\hat{T}\left(v_{n}, z\right), v\right\rangle+\left\langle\hat{T}\left(v_{n}, z\right), v_{n}-v\right\rangle \rightarrow\langle\psi, v\rangle
$$

It suffices to show that

$$
\left\langle\hat{T}\left(v_{n}, z\right), v_{n}-v\right\rangle \rightarrow 0
$$

Now,

$$
\begin{aligned}
\left\langle\hat{T}\left(v_{n}, z\right), v_{n}-v\right\rangle= & \int_{\Omega}\left(\left(\tilde{u}_{n}+x\right) z_{x}+\left(g_{x}-\frac{1}{2}\right) z\right)\left(v_{n}-v\right)_{x}+z_{y}\left(v_{n}-v\right)_{y} \\
= & \int_{\Omega} \sqrt{x} h_{n}(x, y) z_{x}\left(v_{n}-v\right)_{x}+\int\left(g_{x}-\frac{1}{2}\right) z\left(v_{n}-v\right)_{x} \\
& +\int z_{y}\left(v_{n}-v\right)_{y},
\end{aligned}
$$


where $h_{n}$ is a bounded function, since $\bar{u}_{n} \leq(1 / \epsilon) \sqrt{x}$. Now Proposition 4 , with $\beta=1$, shows that $h_{n} \rightarrow h$ in $L^{2}(\Omega)$, and this, along with weak convergence of $v_{n}$ in $\mathcal{H}_{\frac{1}{2}}$, shows that the first integral converges to zero; similarly, the third integral converges to zero by weak convergence of $v_{n}$. In order to estimate the second integral, we write

$$
\int_{\Omega}\left(g_{x}-\frac{1}{2}\right) z\left(v_{n}-v\right)_{x}=\int_{\Omega}\left(g_{x}-\frac{1}{2}\right) x^{3 / 4} \frac{z}{x^{3 / 4}}\left(v_{n}-v\right)_{x}
$$

and again use (26); since $z / x^{3 / 4} \in L^{2}$ and $\left|g_{x}\right|$ is bounded by $x^{-1 / 2}$, the right side of (32) converges to zero, as a consequence of the weak convergence of $v_{n}$ to $v$.

We have proved that the mapping $\hat{T}$ is of calculus of variations type.

By Theorem 10 we now have

Corollary 6 The operator $T$ is pseudo-monotone.

We have now shown that the mapping $T$ is continuous and pseudomonotone. In order to satisfy all the hypotheses of Theorem 7 we have to check that $T$ is coercive.

Theorem 12 The operator $T$ is coercive.

Proof: We shall show that there is a constant $M>0$ such that

$$
\left|B_{g}(v, v)\right| \geq M\|v\|_{\mathcal{H}_{\frac{1}{2}}}^{2}
$$

Recall that

$$
B_{g}(v, v)=\int_{\Omega}(\tilde{u}+x) v_{x}^{2}+\left(g_{x}-\frac{1}{2}\right) v v_{x}+v_{y}^{2} .
$$

We estimate the second integral using the assumptions on $g$. Integrating by parts and using $v=0$ on the boundary, we obtain

$$
\int_{\Omega}\left(g_{x}-\frac{1}{2}\right) v v_{x}=-\int_{\Omega} \frac{1}{2} g_{x x} v^{2} \geq 0
$$

This integral is bounded since $v \in L^{2}(\Omega, \beta)$, for $\beta=3 / 2$. The first integral in (33) can be estimated using the fact that $\bar{u}$ is bounded from below by $\epsilon \sqrt{x}$. Therefore, there exists a $C_{1}>0$ such that

$$
\int_{\Omega}(\bar{u}+x) v_{x}^{2} \geq C_{1} \int_{\Omega} \sqrt{x} v_{x}^{2} .
$$


Combining the last two estimates we obtain

$$
\int_{\Omega}(\tilde{u}+x) v_{x}^{2}+\left(g_{x}-\frac{1}{2}\right) v v_{x} \geq C_{1} \int_{\Omega} \sqrt{x} v_{x}^{2}
$$

Therefore, we have

$$
\left|B_{g}(v, v)\right| \geq M \int_{\Omega} \sqrt{x} v_{x}^{2}+v_{y}^{2}=M\|v\|_{\mathcal{H}_{\frac{1}{2}}}^{2},
$$

where $M=\min \left\{C_{1}, 1\right\}>0$.

By Theorem 9, Corollary 6, and Theorem 12, the operator $T$ defined in Corollary 5 of Theorem 6 is continuous, pseudo-monotone and coercive. Therefore, Theorem 7 gives a weak solution $v$ in the space $\mathcal{H}_{\frac{1}{2}}$.

Theorem 13 For every $f \in \mathcal{H}_{\frac{1}{2}}^{*}$, there exists a function $v \in \mathcal{H}_{\frac{1}{2}}$, such that

$$
B_{g}(v, w)=\int_{\Omega} f w, \quad \text { for all } w \in \mathcal{H}_{\frac{1}{3}} .
$$

In particular, we may now suppose $f$ is given by (15), then the solution of (21), which we have found, is the solution we want, save for the possibility that $B_{g}$ does not coincide with $B$ for our weak solution $v$. We rule this out in the next section.

\subsection{Bounds on the Weak Solution}

In this section we show that a weak solution $v$ of the problem defined by the bivariate form $B_{g}$ satisfies the bounds

$$
\epsilon \sqrt{x} \leq v+g \leq(1 / \epsilon) \sqrt{x} .
$$

In order to show this we construct sub- and super-solutions. We shall show that for every $\delta<\epsilon$ and $M>1 / \epsilon$, the functions $\delta \sqrt{x}-g$ and $M \sqrt{x}-g$ are sub- and super-solutions respectively to (23), where $f$ satisfies (15). This is equivalent to showing that $\delta \sqrt{x}$ and $M \sqrt{x}$ are sub- and super-solutions to

$$
\begin{aligned}
\left((\tilde{u}+x) u_{x}-\frac{1}{2} u\right)_{x}+u_{y y} & =0 & \text { in } \Omega, \\
u & =g & \text { in } \partial \Omega .
\end{aligned}
$$

Here, $\tilde{u}$ is defined by (19), where we set $v+g=u$. 
Theorem 14 The functions $\delta \sqrt{x}$ and $M \sqrt{x}$ are sub- and super-solutions respectively to problem (35), (36).

Proof: If $u<\epsilon \sqrt{x}$ or $u>(1 / \epsilon) \sqrt{x}$, then the equation (35) satisfied by $u$ is linear. Let $w=M \sqrt{x}$; then a calculation shows that $w$ satisfies (35) for any $M$. Since $M \sqrt{x}>g$ on the boundary, $w$ is a super-solution of problem (35), (36). Therefore, any solution $u$ of the problem satisfies $u \leq(1 / \epsilon) \sqrt{x}$, by the comparison principle, Theorem 2. Similarly, we can show that $\delta \sqrt{x}$, for $\delta \leq \epsilon$, is a sub-solution, and therefore $u \geq \epsilon \sqrt{x}$.

Corollary 7 (Existence of a solution to (14)) The weak solution $v$ of Theorem 13 satisfies

$$
\epsilon \sqrt{x} \leq v+g \leq(1 / \epsilon) \sqrt{x} .
$$

and is a solution of

$$
B(v, w)=\int_{\Omega} f w, \quad \forall w \in \mathcal{H}_{\frac{1}{2}}
$$

where the bivariate form $B$ is defined by (16) and $f$ by (15).

\section{Conclusions}

We have proved existence of a weak solution in a weighted Sobolev space $\mathcal{H}_{\frac{1}{2}}$ for problem (3), under assumptions on $g$. By the classical theory of elliptic equations, this weak solution is actually $C^{2}$ everywhere in $\Omega$. Although for uniformly elliptic operators the solution is smooth all the way up to the boundary, we have shown that the degeneracy in this equation induces singular behavior of solutions along the degenerate boundary; in this case, a square-root type singularity.

The growth assumptions on the boundary data $g$ are forced by the need to obtain a coercive operator on the weighted Sobolev space. We believe these assumptions are consistent with the actual behavior of the solution to the original problem. We consider this issue in work now in progress, [3].

Some interesting questions remain open. Our immediate objective is to apply this result to the motivating problem of self-similar solutions to the unsteady TSD equation. To this end, we would like to weaken the hypotheses on $g$; alternatively, we could attempt to formulate the free-boundary problem 
which connects the elliptic and hyperbolic regions in a way that respects these hypotheses, and show that this problem has a solution. In any case, but especially if we adopt this approach, the question of uniqueness remains open.

The theory in this paper also may be extended to unbounded domains $\Omega$ : an appropriate extension for our problern would be to replace the rectangle by a quarter-plane. In this case, the $y$-axis will remain the degenerate boundary. The boundary condition on the $x$-axis might continue to contain upper and lower square-root type bounds on its growth near $x=0$, but a new far-field condition would be needed.

Beyond extensions to the TSD equation, the approach in this paper might be useful in studying other degenerate elliptic equations of Keldysh type. Our interest was focussed on the model problem at hand, but the dependence of the singularity on dimension and on the coefficients of the operator could be studied asymptotically, as in Section 2, and corresponding theorems formulated.

\section{A Appendix: Compactness of the Embed- ding}

Here we assemble the ingredients for the proof of compactness in Theorem 5 in Section 4.2.

The following lemma is a version of the Arzelà-Ascoli theorem for the spaces $L^{2}(\Omega, \beta)$.

Lemma 1 (Adams, [1]) $A$ bounded subset $K \subset L^{2}(\Omega, \beta)$ is precompact in $L^{2}(\Omega, \beta)$ if and only if for every $\epsilon>0$, there exists a $\delta>0$, and a subset $G \subset \subset \Omega$ such that for every $u \in K$ and every $h \in \mathbf{R}^{2}$ with $|h|<\delta$

$$
\begin{gathered}
\int_{\Omega}|u(x+h)-u(x)|^{2} d \mu<\epsilon^{2}, \text { and } \\
\int_{\Omega-G}|u(x)|^{2} d \mu<\epsilon^{2} .
\end{gathered}
$$

Using this Lemma, the following Theorem is also proved in [1, page 33]: 
Theorem 15 Let $K \subset L^{2}(\Omega, \beta)$ be a bounded subset of $L^{2}(\Omega, \beta)$. Suppose that there exists a sequence $\left\{\Omega_{j}\right\}$ of subdomains of $\Omega$ having the following properties:

1. For each $j, \Omega_{j} \subset \Omega_{j+1}$,

2. For each $j$ the set of restrictions to $\Omega_{j}$ of the functions in $K$ is precompact in $L^{2}\left(\Omega_{j}, \beta\right)$,

3. For every $\epsilon>0$ there exists $j$ such that

$$
\int_{\Omega-\Omega_{j}}|u|^{2} d \mu<\epsilon, \quad \forall u \in K .
$$

Then $K$ is precompact in $L^{2}(\Omega, \beta)$.

Using this Theorem we show that the embeddings from Theorem 4 are compact.

Proof of Theorem 5: Continuity of the embedding is proved in Theorem 4 . In order to prove the compactness of the embedding we use Theorem 15 as follows. Let $K$ be a bounded set in $\mathcal{H}_{\alpha}$. Then, there exists a constant $D$ such that for each $u \in K$,

$$
\|u\|_{\mathcal{H}_{\alpha}} \leq D \text {. }
$$

From the continuity of the embedding, it follows that $K$ is bounded in $L^{2}(\Omega, \beta)$.

Let $\Omega_{j} \subset \Omega$ be the subset of $\Omega$ such that $x \geq 1 / j$ for $(x, y) \in \Omega_{j}$. Obviously, $\Omega_{j} \subset \Omega_{j+1}$.

Denote by $\mathcal{H}_{\alpha}\left(\Omega_{j}\right)$ the space of restrictions of functions in $\mathcal{H}_{\alpha}(\Omega)$ to the set $\Omega_{j}$. Define $H^{1}\left(\Omega_{j}\right), L^{2}\left(\Omega_{j}\right)$, and $L^{2}\left(\Omega_{j}, \beta\right)$ the same way. (Here $H^{1}$ is the standard Sobolev space.) The norms in $H^{1}\left(\Omega_{j}\right)$ and $\mathcal{H}_{\alpha}\left(\Omega_{j}\right)$ are equivalent. The same is true for the norms in $L^{2}\left(\Omega_{j}\right)$ and $L^{2}\left(\Omega_{j}, \beta\right)$, for every measure $\mu$ for which $\beta<2-\alpha$. Since $H^{1}\left(\Omega_{j}\right)$ is compactly embedded in $L^{2}\left(\Omega_{j}\right)$, it follows that $\mathcal{H}_{\alpha}\left(\Omega_{j}\right)$ is also compactly embedded in $L^{2}\left(\Omega_{j}, \beta\right), \beta<2-\alpha$.

Therefore, it suffices to show that for every $\epsilon>0$ there exists $j$, such that (37) holds. Let $\epsilon>0$. Assume for the moment that $\beta \neq 1$. Take $j$ such that

$$
\epsilon=\frac{4}{|1-\beta|^{2}} D^{2}\left(\frac{1}{j}\right)^{2-\alpha-\beta} .
$$

Then, using an estimate similar to that in the proof of Theorem 4 with 
$\delta=1-(\alpha+\beta) / 2>0$, we obtain:

$$
\begin{aligned}
\int_{\Omega_{-\Omega_{j}}}|u|^{2} d \mu & \leq \frac{2}{|1-\beta|}\left(\frac{1}{j}\right)^{1-(\alpha+\beta) / 2}\left(\int_{\Omega-\Omega_{j}} \frac{u^{2}}{x^{\beta}} d x d y\right)^{\frac{1}{2}}\left(\int_{\Omega-\Omega_{j}} x^{\alpha} u_{x}^{2}\right)^{\frac{1}{2}} \\
& \leq \frac{2}{|1-\beta|} D\left(\frac{1}{j}\right)^{1-(\alpha+\beta) / 2}\left(\int_{\Omega-\Omega_{j}}|u|^{2} d \mu\right)^{\frac{1}{2}}
\end{aligned}
$$

Therefore,

$$
\int_{\Omega-\Omega_{j}}|u|^{2} d \mu \leq \frac{4}{|1-\beta|^{2}} D^{2}\left(\frac{1}{j}\right)^{2-\alpha-\beta}=\epsilon .
$$

as required. If $\beta=1$, one modifies the choice of $\epsilon$ in (38).

Acknowledgments: It is a pleasure to mention the hospitality of the Fields Institute in Waterloo, Canada. The research reported in this paper was initiated during an extended visit by both authors from January to June, 1993. We benefited from helpful discussions with Chris Cosner and Gary Lieberman. We thank David Wagner, who first brought this problem to our attention. We also thank Cathleen Morawetz for several discussions and much encouragement, and thank her and Esteban Tabak and Reuben Rosales for making their preprints available to us.

A discussion with Joe McKenna at the Fields Institute led to an independent investigation of a problem similar to the boundary value problem for equation (3): Choi, Lazer and McKenna, [4], consider a family of equations which includes a singular term of the form $u u_{x x}+u_{y y}$ but a different lower order term. They use a comparison principle and a priori estimates to bound the solution and then use compactness arguments to show existence in a bounded, convex domain with a smooth boundary. The solution they obtain has the same type of square root singularity.

\section{References}

[1] Robert A. Adams, Sobolev Spaces, Academic Press, New York, 1975.

[2] M. Brio and J. K. Hunter, 'Mach reflection for the two dimensional Burgers equation', Physica D 60, 1992, 194-207. 
[3] S. Čanić, B. L. Keyfitz, and D. H. Wagner, 'A bifurcation diagram for oblique shock interactions in the unsteady transonic small disturbance equation', Proceedings of the Fifth International Conference on Hyperbolic Problems, Theory, Numerics, and Applications, to appear.

[4] Y. S. Choi, A. C. Lazer and P. J. McKenna, 'On a singular quasilinear elliptic boundary value problem', Proc. Amer. Math. Soc., in press.

[5] P. Colella and L.F. Henderson, "The von Neumann paradox for the diffraction of weak shock waves', J. Fluid Mech. (1990), 213, 71-94.

[6] D. Gilbarg and N. S. Trudinger, Elliptic Partial Differential Equations of Second Order, Springer, New York, 1977.

[7] J. K. Hunter, 'Nonlinear geometrical optics', in Multidimensional Hyperbotic Problems and Computations, (ed. J. Glimm and A. Majda) IMA Vol 29, Springer, New York, 1991, 179-197.

[8] M. V. Keldysh, 'On some cases of degenerate elliptic equations on the boundary of a domain', Doklady Acad. Nauk USSR, 77, 1951, 181-183 (in Russian).

[9] J. J. Kohn and L. Nirenberg, 'Degenerate elliptic-parabolic equations of second order', Comm. Pure Appl. Math., 20, 1967, 797-872.

[10] C. S. Morawetz, 'Potential theory for regular and Mach reflection of a shock at a wedge', Comm. Pure Appl. Math., 1994, to appear.

[11] J. Nečas, Introduction to the Theory of Nonlinear Elliptic Equations, John Wiley \& Sons, New York, 1986.

[12] O. A. Oleỉnik and E. V. Radkevič, Second Order Equations with Nonnegative Characteristic Form, (trans. P. Fife), American Math. Soc., Providence, 1973.

[13] M. Renardy and R. C. Rogers, An Introduction to Partial Differential Equations, Springer, New York, 1993.

[14] R. Rosales, 'An introduction to weakly nonlinear geometrical optics', in Multidimensional Hyperbolic Problems and Computations, (ed. J. Glimm and A. Majda) IMA Vol 29, Springer, New York, 1991, 281-310. 
[15] A. Sakurai, L.F. Henderson, K. Takayama, Z. Walenta, P. Colella, 'On the von Neumann paradox of weak Mach reflection', Fluid Dynam. Research , 4 , 1989, 333-346.

[16] E. G. Tabak and R. R. Rosales, 'Weak shock focusing and the von Neumann paradox', Physics of Fluids A, to appear.

[17] H. Triebel, Interpolation Theory, Function Spaces, Differential Operators, North-Holland, Amsterdam, 1.978. 